\title{
Análise de Desempenho de uma Ferramenta para Visualização de Hashes em Dispositivos Móveis
}

\author{
Henrique Araújo de Carvalho, Jorge Miguel Ribeiro, \\ Daniel Macêdo Batista, José Coelho de Pina
}

${ }^{1}$ Departamento de Ciência da Computação

Instituto de Matemática e Estatística

Universidade de São Paulo (USP)

\author{
henriquecarvalho@usp.br, jorgemiguelribeiro92@gmail.com \\ \{batista, coelho\}eime.usp.br
}

\begin{abstract}
A security application that enhances communication privacy must have a performance that ensures its usability without compromising the reservation of scarce resources, especially on mobile devices. Among such security applications, those such as hashify stand out, which manipulate or generate animations from cryptographic hashes. This paper presents the performance analysis of hashify, measuring the time taken to draw animations and evaluating memory and network consumption. Preliminary results obtained from an Android device showed that the images generated by the software are drawn, on average, in 22.6ms, a relatively low time showing that hashify can be integrated into mobile applications.
\end{abstract}

Resumo. Um aplicativo de segurança que aumente a privacidade de comunicações deve ter desempenho que garanta a sua usabilidade sem comprometer a reserva de recursos escassos, principalmente em dispositivos móveis. Dentre esses aplicativos, destacam-se aqueles, como o hashify, que manipulam ou geram animações a partir de hashes criptográficos. Este artigo apresenta a análise de desempenho do hashify, avaliando o tempo de geração de animações e consumo de memória e rede. Resultados preliminares obtidos com um dispositivo Android mostraram que a geração de imagens pelo software ocorre, em média, em 22,6ms, um tempo relativamente baixo e que mostra que ele pode vir a ser integrado em aplicativos móveis.

\section{Introdução}

A comparação de hashes criptográficos é uma atividade cotidiana em segurança da informação e geralmente é atribuída a sistemas automatizados. Entretanto, muitas vezes, a garantia de segurança das comunicações pela Internet depende da comparação desses hashes por pessoas. Isso ocorre, por exemplo, na comparação de chaves criptográficas em aplicativos de mensagens e na comparação de certificados digitais de websites. Este trabalho realiza o exame de uma ferramenta $\left.\right|^{1}$ que facilita a comparação desses hashes por

\footnotetext{
${ }^{1} \mathrm{O}$ código da ferramenta, chamada hashify, foi disponibilizado sob a licença MIT e está disponível em https://gitlab.com/jorgemiguelribeiro92/hashify/
} 
pessoas, avaliando a viabilidade de sua utilização e de sua implementação em aplicativos Android.

O restante deste artigo está organizado da seguinte forma: A Seção 2 resume os trabalhos relacionados e apresenta a motivação para este artigo. A Seção 3 resume o funcionamento do hashify. A Seção 4 descreve a metodologia utilizada na análise de desempenho. A Seção 5 apresenta os resultados obtidos. O artigo é finalizado com a apresentação das conclusões e dos próximos passos na Seção 6 .

\section{Trabalhos Relacionados e Motivação}

Ao longo das últimas duas décadas, vários estudos propuseram e examinaram ferramentas com a finalidade de facilitar a comparação de hashes criptográficos por pessoas, que são reconhecidamente ruins nessa tarefa [Perrig and Song 1999]. Enquanto nesses estudos os autores encontraram dificuldades na adoção de um sistema de geração de hashes visuais, a ferramenta hashif $y^{2}$ busca superar essas dificuldades, avançando o estado da arte na comparação de hashes criptográficos por meio da geração de animações de 2 segundos de duração [Ribeiro et al. 2020].

Por exemplo, em Hash Visualization: a New Technique to improve Real-World Security (1999) [Perrig and Song 1999], os autores propõem a utilização de visualização de hashes como uma primeira etapa de verificação de senhas, feita pelos usuários, e como validação de chaves criptográficas. Os autores propõem a utilização de um algoritmo desenvolvido por Andrej Bauer, conhecido como Random Art, para a criação de hashes visuais. $\mathrm{O}$ algoritmo usa uma string de bits como a semente para um gerador de números aleatórios, que constrói uma expressão que descreve a função que irá gerar a imagem, mapeando cada pixel a um valor.

O hashify representa uma evolução desta ideia, já que propõe a utilização de imagens de objetos para uso na comparação de chaves criptográficas, e não imagens geradas de forma caótica. Supõe-se ser mais fácil comparar objetos e cores a comparar imagens genéricas, que podem envolver diferentes contrastes e posições de linhas.

Em Developing and testing SCoP - a visual hash scheme (2014) [Maina Olembo et al. 2014], os autores desenvolveram e testaram SCoP, uma proposta de hash visual que poderia substituir a comparação de sequências alfanuméricas. Os esquemas propostos nesse estudo, submetidos a experimentos com pessoas, foram mal sucedidos se consideradas outras limitações para a utilização de hashes visuais no mundo real, indicando que seria mais difícil ainda de se comparar hashes visuais em situações em que usuários não têm acesso à imagem correta e apenas lembram dela.

No caso do hashify, propõe-se a sua utilização em conjunto com um método de certificação alternativo, como um smartphone, de modo que não seria necessário lembrar exatamente do resultado visual do hash.

Em Can Unicorns Help Users Compare Crypto Key Fingerprints? (2017) [Tan et al. 2017] os autores desenvolvem um experimento realizado com 661 pessoas, que envolve a simulação de um ataque Man in the Middle (MitM). Foi solicitado às pessoas para compararem hashes que foram sutilmente modificados, à semelhança de como

\footnotetext{
${ }^{2}$ Uma demonstração de funcionamento do hashify pode ser vista em https://youtu.be/ raFkfRRXBM0
} 
um atacante poderia agir para interceptar comunicações. Os autores concluem que as representações gráficas tiveram um sucesso inconclusivo, enquanto representações textuais foram mais bem sucedidas na comparação de hashes criptográficos. Isso porque representações gráficas de hashes, em geral, estariam mais suscetíveis a terem detalhes e aspectos essenciais ignorados na comparação, apesar de permitirem comparações mais rápidas e fáceis.

Ao contrário dos algoritmos usados por este estudo, o hashify é objetivamente mais fácil de se comparar porque envolve figuras, com cores evidentes, movimentos específicos e contendo um pequeno texto, o que também ajuda na orientação de pessoas sobre como se deve comparar hashes visuais.

Portanto, o hashify é uma alternativa bem sucedida às demais ferramentas que se propõem a facilitar a comparação de hashes criptográficos. Resta analisar o desempenho do hashify em dispositivos móveis, com a finalidade de verificar sua viabilidade como uma ferramenta de segurança nesses dispositivos. Essa análise é importante pelo fato dos dispositivos possuírem baterias como suas fontes principais de energia, o que justifica estudar o impacto que a ferramenta tem no consumo de recursos que possa levar a um consumo acelerado da bateria. Esse tipo de preocupação, relacionado à "complexidade energética" de algoritmos e componentes de software, tem sido discutida nos últimos anos na literatura especializada [Roy et al. 2013][ [Jain et al. 2005].

Este artigo apresenta os resultados preliminares alcançados na análise de desempenho do hashify. São avaliados tempo de geração das animações e consumo de memória e rede. Os resultados obtidos mostram que, a princípio, o hashify pode vir a ser integrado em aplicativos móveis. Por exemplo, em média, o tempo necessário para geração de animações foi de 22,6ms em um smartphone Samsung S20 FE, um dispositivo lançado no mercado em Outubro de 2020.

\section{Funcionamento do hashify}

O funcionamento do hashify é baseado em um algoritmo de geração de imagens com animações únicas derivadas a partir da composição de funções de hash tendo como argumento inicial o hash original. A Figura 1 mostra uma sequência com 5 quadros de uma animação gerada pelo hashify.

Na versão atual, o hashify pode ser utilizado embutido como uma extensão do navegador Firefox, o que permite que o mesmo seja utilizado em dispositivos móveis sem necessidade de alteração do seu código-fonte.

\section{Metodologia}

Um aplicativo de segurança que aumente a privacidade de comunicações deve ter desempenho que garanta a sua usabilidade sem comprometer a reserva de recursos escassos, principalmente em dispositivos móveis. Por este motivo, o presente estudo analisa, de forma estruturada, o desempenho do hashify, implementado como extensão no navegador Firefox, em execução em um smartphone.

A análise de desempenho foi realizada por meio da coleta de rastros. Rastros são arquivos que guardam informações sobre os processos executados no sistema operacional. Esses rastros contêm informações sobre eventos que ocorreram durante a execução de 


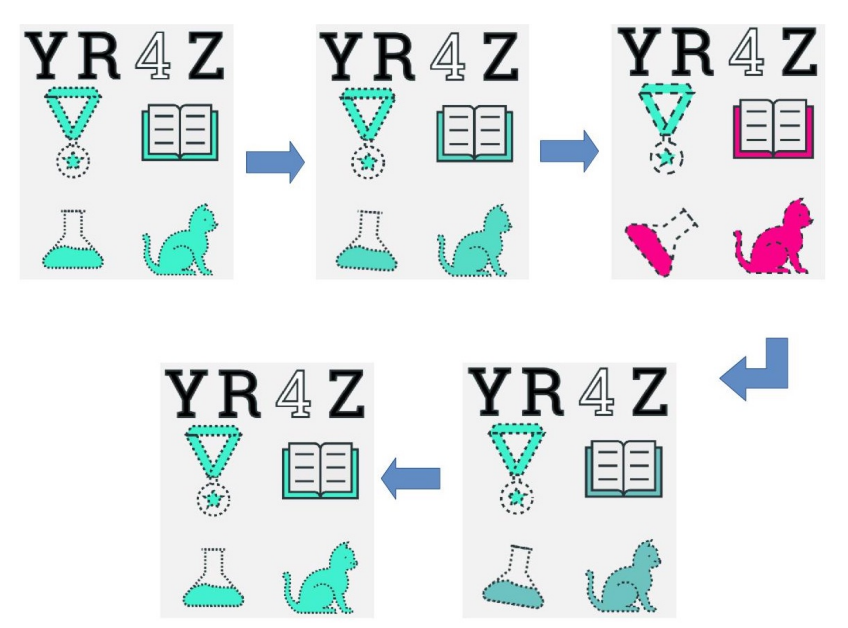

Figura 1. Quadros da animação gerada pelo hashify para o hash e0d31d7599daebee65a15a636736d65dae6963d2.

uma aplicação. Os eventos são associados a uma trilha específica, que funciona como uma linha do tempo crescente monotonicamente. Uma trilha corresponde a uma sequência independente de execução, como uma única thread em um processo [Google 2021b].

Os tipos de eventos analisados foram fatias e contadores. Fatias cobrem o período de tempo desde a chamada de uma função até seu término. Contadores são valores numéricos variáveis mostrados em cada instante de tempo.

A coleta dos rastros foi realizada com o hashi fy, instalado como uma extensão do Firefox, aberto em um smartphone e com uma única interação com o smartphone: um clique no botão de gerar animação. Todas as coletas de rastros foram realizadas em um Samsung modelo Galaxy S20 FE, exynos990, equipado com o Sistema Operacional Android 11 e com Firefox Browser Nightly 92.0a1.

Para a coleta dos rastros, foi usado o aplicativo Rastreamento de sistema, executado no próprio Android, que salva a atividade do dispositivo em um arquivo de rastreamento no formato perfetto-trace [Google 2020b]. Para a visualização e organização destes rastros, foi usada a ferramenta Perfetto [Google 2021a]. Além de permitir a visualização dos dados por meio de uma linha do tempo, o Perfetto exibe o tempo tomado pelos eventos de uma thread em uma tabela. Todos os dados quantitativos foram coletados dessa tabela. Usando essas ferramentas, foram coletados 25 rastros que permitiram uma análise estatística de informações sobre tempo de geração das animações, consumo de memória e consumo de rede.

Para que se possa comparar o desempenho do hashify a uma aplicação similar, também foram coletados 5 rastros durante a execução do software RoboHash [Davis 2011], uma aplicação com funcionamento similar ao do hashify.

\section{Análise de Desempenho}

Nesta seção, avalia-se o desempenho do hashify por meio dos rastros coletados. Esse desempenho foi medido através do tempo de geração de quadros, do consumo de memória e do consumo de rede. Ao final de cada uma das próximas subseções, o desempenho do hashify foi comparado com o desempenho do software RoboHash. 


\subsection{Tempo de Geração de Quadros}

Um baixo tempo de geração de quadros é essencial para que uma aplicação de dispositivo móvel não tenha uma taxa de quadros instável ou para que o usuário não perceba uma alta latência da aplicação, o que é conhecido por instabilidade. Para que não haja instabilidade, a documentação do Android sugere que a geração de imagens e sua composição sejam feitas em um intervalo de $16 \mathrm{~ms}$, ou seja, os quadros precisam ser gerados e compostos a uma frequência de aproximadamente $60 \mathrm{~Hz}$. Caso a geração de quadros não aconteça a tempo, é possível que o desenho seja atrasado em alguns milissegundos. É deste atraso que vem a instabilidade experimentada por um usuário [Google 2020a].

Para saber avaliar o desempenho da aplicação neste quesito, antes de visualizar os dados do tempo de geração de quadros, é preciso entender o comportamento das threads responsáveis pela geração de quadros no Android.

A UI Thread (thread da interface do usuário) é a thread responsável pela geração e composição de imagens. É essa thread que recebe e avalia entrada, desenha a interface e organiza a execução de outros eventos de tela. Em geral, é a thread principal de um aplicativo Android. A RenderThread tem a função de rodar computações da GPU. Ela é responsável pela renderização de animações. A thread SurfaceFlinger é responsável por consumir as superfícies desenhadas por outros processos e compô-las em um único quadro a ser mostrado na tela. Finalmente, o Choreographer é o evento da UI Thread responsável por coordenar o tempo dessas threads.

A Figura 2 permite visualizar o trabalho das threads descritas acima. O evento Choreographer (linha em marrom na figura) é chamado na UI Thread (identificada como "AndroidUI" à esquerda na figura) e, então, o trabalho da CPU se inicia na construção dos quadros até quando a RenderThread é chamada para renderizar as imagens. Como pode ser visto na figura, ambas as threads são chamadas duas vezes seguidas, de modo que consideramos o final da segunda vez para o marco do término da geração dos quadros. Nesta amostra, tudo tem duração aproximada de 19,2ms.

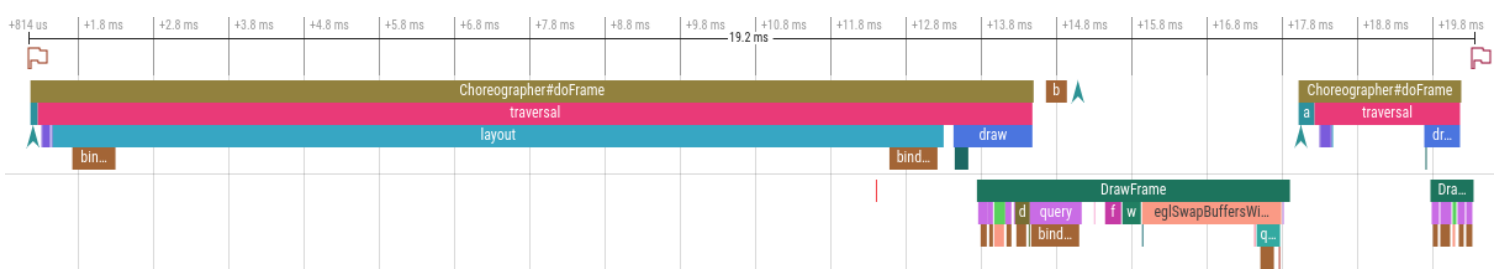

Figura 2. Linha do tempo de execução (Saída do software Perfetto)

Apesar dessa amostra ter uma composição que demora aproximadamente 3,2ms a mais que o limite sugerido de $16 \mathrm{~ms}$, entendemos que isso não seria suficiente para que o usuário sinta a instabilidade. Isso porque o atraso ocorre durante um único quadro e a frequência de geração de quadros, a um tempo de aproximadamente $20 \mathrm{~ms}$, é de $50 \mathrm{~Hz}$ e continua alta e, possivelmente, indetectável por pessoas. Assim, entendemos que o hashify tem um desempenho de tempo de geração de quadros satisfatório se ele se mantiver abaixo de 20ms. Pelos dados coletados (Figura 3), foi possível verificar que isso ocorre em pelo menos $50 \%$ das vezes, uma vez que a mediana da duração da geração de quadros foi $19,2 \mathrm{~ms}$. A média da duração foi $22,6 \mathrm{~ms}$ para os dados obtidos, com um 
desvio padrão de 6,4ms. Ou seja, em geral o hashify performa muito bem em tempo de geração de quadros e não deve atrapalhar a experiência do usuário com instabilidade.
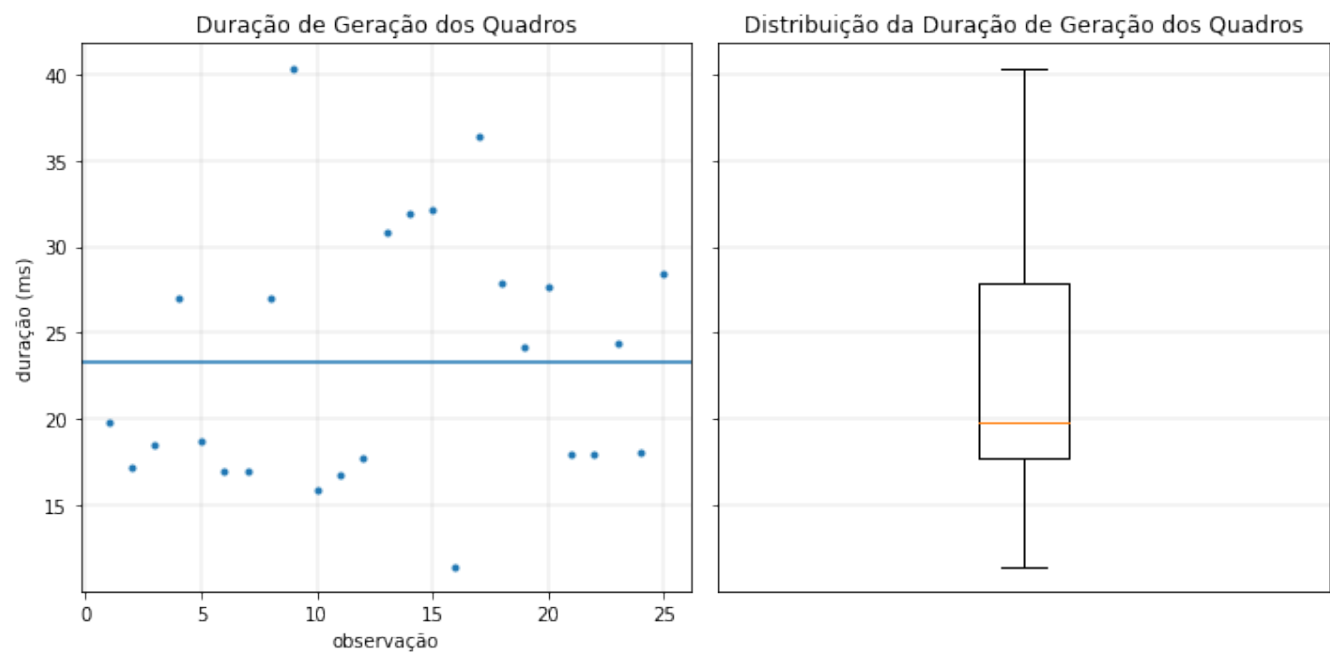

Figura 3. Observações do tempo de execução

A título de comparação, o RoboHash levou em média 28ms para gerar uma imagem, o que significa que o hashify possui desempenho médio, em termos de geração de quadros, cerca de 19,3\% melhor que o deste software.

\subsection{Consumo de Memória}

Alguns contadores presentes no rastro são capazes de dar uma ideia sobre o consumo de memória pelo hashify. A Figura 4 permite visualizar os dados de memória que são mostrados pelo rastro. Esse corte do rastro foi feito exatamente durante a geração dos quadros pelo hashify.

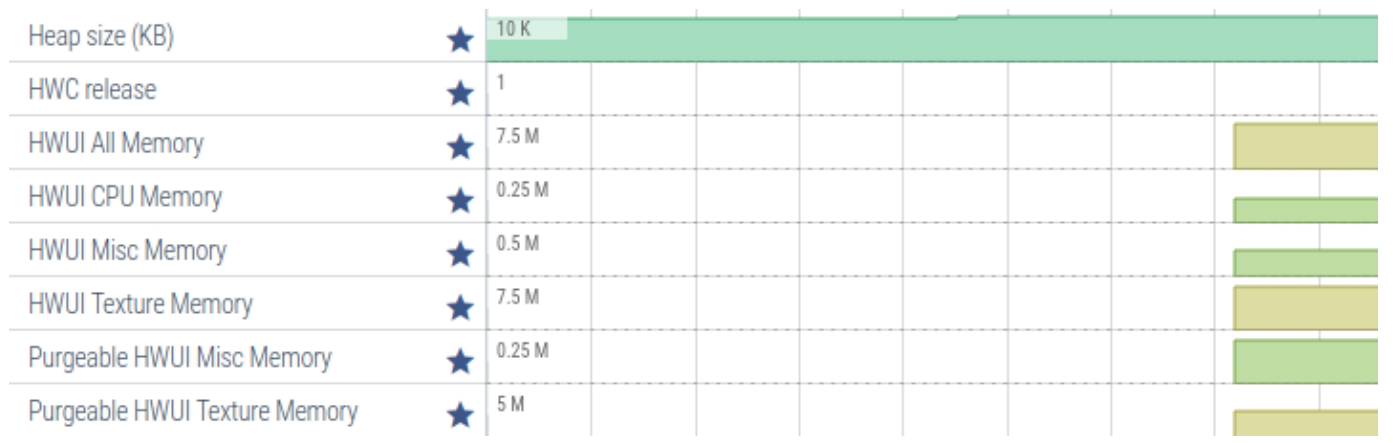

Figura 4. Consumo de memória (Saída do software Perfetto)

Pode-se notar que o rastro dispõe duas memórias diferentes para a execução da aplicação, o heap e a memória HWUI. Para esta última, além do contador que conta a memória total HWUI, existem outros contadores que mostram a memória HWUI segmentada.

Enquanto a memória do heap mede a memória alocada na RAM durante a execução da aplicação, memória total HWUI é a soma de toda a memória alocada para caches (cache de textura, de camadas, de gradiente, etc) da biblioteca Android HWUI, 
que permite que componentes da interface do usuário sejam acelerados pela GPU. É por este motivo que no rastro da figura o contador dessa memória sobe imediatamente após o evento DrawFrame, da RenderThread, terminar seu trabalho. Portanto, para esta análise interessa a memória do heap e a memória total HWUI.

Sobre a memória do heap, interessa para a análise a variação do tamanho dessa memória. Considera-se variação de memória do heap como a diferença entre o valor anterior e o valor posterior à geração de quadros. Esses dados foram dispostos na Figura 5 . Neste caso, a mediana das observações é bem próxima da média, o que indica que a variação da memória do heap tem uma distribuição normal. Em média existe um consumo de aproximadamente $82,7 \mathrm{~KB}$ de memória do heap, com um desvio padrão de 23,0KB, e espera-se que, em pelo menos $50 \%$ das vezes, seja consumido $80,0 \mathrm{~KB}$ ou menos.
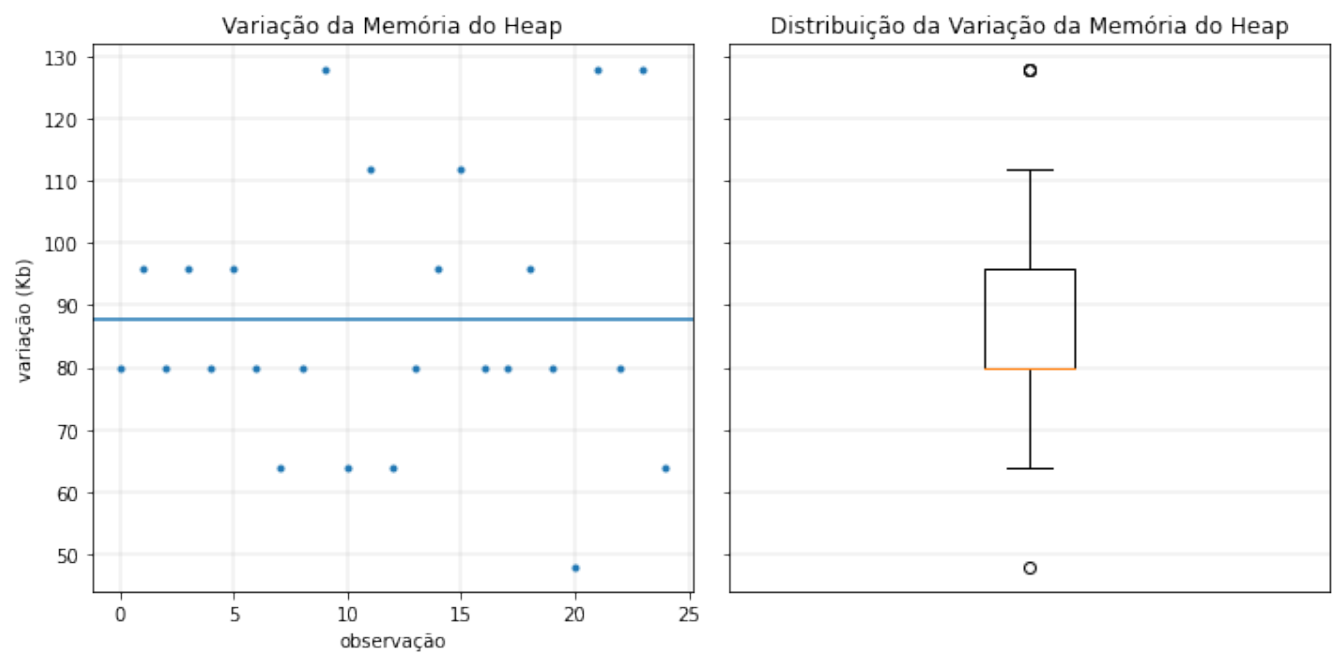

Figura 5. Observações do consumo de memória do heap

Sobre a memória total HWUI, interessa para a análise o tamanho dessa memória após o evento DrawFrame terminar. Esse valor é constante para todas as execuções, 7,371860MB. A título de comparação, após a execução do software RoboHash, a memória total HWUI tem valor de 4,365162MB. Embora o consumo do hashify seja maior, vale observar que a ferramenta gera uma animação e não uma imagem estática.

\subsection{Consumo de Rede}

NetworkPolicy é o processo que chama um conjunto de eventos de rede. Entretanto, não foi identificado qualquer consumo de rede pelo hashify, de modo que é possível criar animações com a extensão mesmo sem acesso à Internet.

Este resultado era esperado, uma vez que a extensão foi carregada localmente e executada no próprio smartphone. De toda forma, a análise se faz necessária, diante da possibilidade das bibliotecas usadas no desenvolvimento da aplicação poderem acessar recursos de rede. Ainda, uma vez que o RoboHash está acessível unicamente por seu website, não foi possível realizar a comparação deste quesito entre as duas aplicações.

\section{Conclusão e Próximos Passos}

Este artigo apresentou a análise de desempenho do hashify, uma aplicação de geração de animações a partir de hashes, com a finalidade de avaliar a viabilidade de sua 
implementação em aplicativos de segurança em dispositivos móveis.

Após análise preliminar de rastros obtidos durante a execução do software, conclui-se que o software possui bom desempenho durante a geração das animações e que é, portanto, uma alternativa bem sucedida às demais ferramentas que propuseram a facilitar a comparação de hashes criptográficos no passado. Dessa forma, entende-se que o hashify pode vir a ser integrado em aplicações de dispositivos móveis sem comprometer o desempenho dessas aplicações. O trabalho encontra-se em andamento. Como próximos passos, pretende-se analisar o consumo de bateria do hashify, repetir os experimentos em pelo menos outro modelo de smartphone e realizar experimentos com pessoas, a fim de testar a eficácia do hashify.

\section{Agradecimentos}

Esta pesquisa é parte do INCT da Internet do Futuro para Cidades Inteligentes, financiado por CNPq (proc. 465446/2014-0), Coordenação de Aperfeiçoamento de Pessoal de Nível Superior - Brasil (CAPES) - Código de Financiamento 001 e FAPESP (procs. 14/509371 e 15/24485-9). Também é parte dos projetos FAPESP proc. 18/22979-2, 18/23098-0 e 21/04266-1.

\section{Referências}

Davis, C. (2011). RoboHash. https : / robohash.org/. Acessado em 23/07/2021.

Google (2020a). Renderização lenta. https://developer.android.com/ topic/performance/vitals/render. Acessado em 22/07/2021.

Google (2020b). Visão geral do rastreamento do sistema. https://developer. android.com/topic/performance/tracing. Acessado em 12/07/2021.

Google (2021a). Perfetto - System profiling, app tracing and trace analysis. https: //perfetto.dev/. Acessado em 12/07/2021.

Google (2021b). Track events (Tracing SDK). https://perfetto.dev/docs/ instrumentation/track-events. Acessado em 22/07/2021.

Jain, R., Molnar, D., and Ramzan, Z. (2005). Towards a Model of Energy Complexity for Algorithms [Mobile Wireless Applications]. In IEEE Wireless Communications and Networking Conference, 2005, volume 3, pages 1884-1890.

Maina Olembo, M., Kilian, T., Stockhardt, S., Hülsing, A., and Volkamer, M. (2014). Developing and Testing SCoP-a Visual Hash Scheme. Information Management \& Computer Security, 22(4):382-392.

Perrig, A. and Song, D. (1999). Hash Visualization: a New Technique to Improve RealWorld Security. In CryTEC '99.

Ribeiro, J. M., Batista, D. M., and de Pina, J. C. (2020). hashify: Uma Ferramenta para Visualização de Hashes com Animações. In Anais do Salão de Ferramentas do SBSeg.

Roy, S., Rudra, A., and Verma, A. (2013). An Energy Complexity Model for Algorithms. In 4th Conference on Innovations in Theoretical Computer Science, page 283-304.

Tan, J., Bauer, L., Bonneau, J., Cranor, L. F., Thomas, J., and Ur, B. (2017). Can Unicorns Help Users Compare Crypto Key Fingerprints? In ACM CHI'17, pages 3787-3798. 